\title{
DISERTACIONES
}

ESTUDIOS

Para citar este artículo: Tsaousi, A. (2018). El disfrute de la experiencia audiovisual por personas sordas y con diversidad auditiva: la representación visual de los efectos sonoros. Anuario Electrónico de Estudios en Comunicación Social "Disertaciones", 11(1), 110-126. Doi: http://dx.doi.org/10.12804/revistas.urosario. edu.co/disertaciones/a.4982

\section{EL DISFRUTE DE LA EXPERIENCIA AUDIOVISUAL POR PERSONAS SORDAS Y CON DIVERSIDAD AUDITIVA: LA REPRESENTACIÓN VISUAL DE LOS EFECTOS SONOROS*}

\section{The Enjoyment of the Audiovisual Experience by Deaf and Hearing-Impaired People: Visual Representation of Sound Effects \\ O disfrute da experiência audiovisual por pessoas surdas e com diversidade auditiva: a representação visual dos efeitos sonoros}

Aikaterini Tsaousi, Universitat Autónoma de Barcelona

katetsaou@gmail.com

Recibido: 04 de julio de 2016

Aceptado: 07 de noviembre de 2016

\section{RESUMEN}

El objetivo del presente estudio es observar la experiencia narrativa de los receptores que usan subtítulos para sordos y/o con dificultades de audición en el consumo de productos audiovisuales. Concretamente el estudio busca determinar si existe un efecto en el disfrute y en algunos de sus principales componentes dependiendo de

* Este estudio de investigación forma parte del proyecto FFI2012-39056-C02-01 "Subtitling for the Deaf and Hard of Hearing and Audio Description: New Formats", financiado por el Ministerio de Economía y Competitividad del Gobierno español y del proyecto Europeo HBB4ALL FP7 CIP-ICT-PSP.2013.5.1 \# 621014. Además, está financiado por los fondos del gobierno Catalán 2009SGR700 y FI-DGR 2014. 


\section{DISERTACIONES}

ESTUDIOS

Estudios de audiencias y recepción: audiencias minoritarias y nuevas mediaciones

ISSN: 1856-9536

Doi: http://dx.doi.org/10.12804/revistas.urosario.edu.co/disertaciones/v11i1

Volumen 11, Número 1 / Enero-junio 2018

Versión PDF para imprimir desde

http://revistas.urosario.edu.co/index.php/disertaciones

si en pantalla se usan representaciones verbales o no verbales de los efectos sonoros que acompañan la narrativa audiovisual. Un total de 46 personas fueron asignadas al azar a una de dos condiciones experimentales: ver películas con subtítulos o con íconos. Tras el visionado, los participantes evaluaron su disfrute y enganche narrativo mediante escalas de Likert. Los análisis estadísticos muestran que no existe un efecto significativo del modo de presentación en el disfrute, agrado, inclinación a repetir la experiencia y enganche. Basándonos en modelos desarrollados por los estudios de comunicación y de psicología de los medios para medir conceptos vinculados a la experiencia de los usuarios, este manuscrito explora las posibilidades de aplicar esas medidas en el campo de la recepción de audiencias desde los estudios de traducción audiovisual y accesibilidad mediática.

Palabras clave: subtitulado para sordos, etiquetaje de sonido, experiencia narrativa, disfrute, compromiso narrativo.

\section{ABSTRACT}

The goal of this study is to observe the narrative experience of viewers with hearing difficulties and those who use subtitles for the deaf in the consumption of audiovisual products. Specifically, the study seeks to determine if there is an effect on enjoyment and some of its main components, depending on the use of either verbal or nonverbal representations of sound effects that accompany an audiovisual narrative. A total of 46 viewers were randomly assigned to one of two experimental conditions: viewing films with subtitles or with icons. After viewing, participants assessed their enjoyment and narrative engagement using Likert scales. Statistical analyses show that there is no significant effect of the mode of presentation on enjoyment, inclination to repeat the experience, or engagement. Based on models developed by communication studies and media psychology to measure concepts related to user experience, this manuscript explores the possibilities of applying these measures from audiovisual translation studies and media accessibility to the field of audience reception.

Keywords: Subtitling for the deaf, sound labeling, narrative experience, enjoyment, narrative engagement.

\section{RESUMO}

O Objetivo do presente estudo é observar a experiência narrativa dos receptores que usam subtítulos para surdos e/ ou com dificuldades de audição no consumo de produtos audiovisuais. Concretamente, o estudo busca determinar se existe um efeito no disfrute e em alguns de seus principais componentes dependendo de se na tela se usam representações verbais ou não verbais dos efeitos sonoros que acompanham à narrativa audiovisual. Um total de 46 pessoas foram assignadas ao acaso a uma de duas condições experimentais: assistir filmes com subtítulos ou ícones. Após assistir, os participantes avaliaram seu disfrute e ligação narrativa mediante escalas de Likert. As análises estatísticas mostram que não existe um efeito significativo do modo de apresentação no disfrute, agrado, inclinação a repetir a experiência e ligação. Baseando-nos em modelos desenvolvidos pelos estudos de comunicação e de psicologia dos meios para medir 


\section{DISERTACIONES}

ESTUDIOS

Estudios de audiencias y recepción: audiencias minoritarias y nuevas mediaciones

ISSN: 1856-9536

Doi: http://dx.doi.org/10.12804/revistas.urosario.edu.co/disertaciones/v11i1

Volumen 11, Número 1 / Enero-junio 2018

Versión PDF para imprimir desde

http://revistas.urosario.edu.co/index.php/disertaciones

conceitos vinculados à experiência dos usuários, este manuscrito explora as possibilidades de aplicar essas medidas no campo da recepção de audiências desde os estudos de tradução audiovisual e acessibilidade mediática.

Palavras-chave: subtítulos para surdos, etiquetagem de sonido, experiência narrativa, disfrute, compromisso narrativo.

\section{Introducción: Estudios de recepción y traducción audiovisual}

La traducción audiovisual (TAV) es una de las disciplinas más recientes dentro de los estudios de traducción que examina, entre otros temas, la recepción de narrativas por parte de los espectadores (Gambier, 2006; Kruger et al., 2017). La recepción en tav se relaciona con los cambios en el código lingüístico y con la transferencia intersemiótica (Gottlieb, 2005). Dicha transferencia es especialmente pertinente en el ámbito de la accesibilidad a los medios de comunicación, que incluye prácticas como el subtitulado para sordos o personas con diversidad funcional auditiva (SPS), y la audiodescripción (AD) para personas invidentes o con diversidad funcional visual.

En el caso del sps, los subtítulos transmiten de forma visual la información sonora, aunque no se consideran equivalentes ya que transmiten mediante diferentes canales (visual vs. auditivo). Desde el punto de vista funcional, el objetivo principal del sps reside en minimizar el esfuerzo a la hora de procesar la información sin restar el disfrute de la experiencia audiovisual. Según Neves $(2008)^{1}$

los subtítulos no deberían obstruir el disfrute, ya que la experiencia de ver un programa televisivo o una película en el cine no debería centrarse en leer los subtítulos, sino, al contrario, en olvidarse de que están presentes y vivir la experiencia audiovisual en su totalidad (s.p.).

Por este motivo, los primeros estudios empíricos en este ámbito se enfocaron en el procesamiento de la información por parte de los espectadores con diversidad auditiva (Arnáiz-Uzquiza, 2012; Perego, 2012; Romero-Fresco, 2015). El procesamiento incluye tanto la lectura de los subtítulos como la distribución de la atención en el resto de la imagen, por lo que el método más usado por esos estudios es el seguimiento de los movimientos oculares. Dicho método ha ofrecido muchos datos a nivel perceptual sobre el comportamiento de los espectadores cuando procesan textos audiovisuales con subtítulos (intralingüísticos e interlingüísticos)2. Por otra parte, la mayoría de estos estudios han usado una metodología mixta, combinada con otras medidas como la comprensión del texto audiovisual, la memoria, la actitud o las preferencias de los espectadores (Neves, 2005; Cambra et al., 2008; Kirkland, 1999; Lorenzo, 2010). Considérese, sin embargo, que la medición de la actitud de los espectadores es menos directa que la de los movimientos oculares, dado que es inferencial (Henerson, Morris \& Firz-Gibbon,

1 Del original en inglés: "[s]ubtitles should never be in the way of enjoyment. Watching television, going to the movies or attending a live performance is not about reading subtitles, it is all about forgetting they are there and taking in the whole audiovisual experience as one" (traducción de la autora).

2 La lista de estudios que emplean este método está en constante aumento: Szarkowska et ál. (2011), Arnáiz-Uzquiza (2012), Orero \& Vilaró (2012), Perego (2012), Rajedran et ál. (2013), Kruger \& Steyn (2014), Miquel-Iriarte (2014), Redmond (2015), Romero-Fresco (2015).

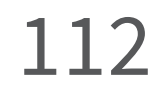




\section{DISERTACIONES}

ESTUDIOS

Estudios de audiencias y recepción: audiencias minoritarias y nuevas mediaciones

ISSN: 1856-9536

Doi: http://dx.doi.org/10.12804/revistas.urosario.edu.co/disertaciones/v11i1

Volumen 11, Número 1 / Enero-junio 2018

Versión PDF para imprimir desde

http://revistas.urosario.edu.co/index.php/disertaciones

1987, p. 13). Por lo tanto, los métodos empleados en tav son, a menudo, mixtos e incluyen asimismo medidas de cuestionarios y entrevistas (Pérez-González, 2014).

Ahora bien, los últimos años han sido testigos de un incremento en los trabajos experimentales para explorar las respuestas a las narrativas. A los métodos descritos se han añadido los instrumentos fisiológicos de medición, para observar el ritmo cardiaco o la actividad electrodermal (Ramos, 2015), aunque este último método todavía no está consolidado en el campo. Por otro lado, la medición de actitudes mediante cuestionarios y entrevistas se ha desarrollado para incluir el estudio de conceptos complejos provenientes de la disciplina de la psicología de los medios (media psychology), como la presencia o la transportación de los espectadores mientras están inmersos en una narrativa audiovisual (Jennett et al., 2008; Kruger et al., 2015). Por ahora, estos trabajos tempranos se centran en observar la AD (Fryer \& Freeman, 2012). Los conceptos más populares en las nuevas investigaciones están ligados a uno de los objetivos principales de los medios de comunicación y del cine: el entretenimiento (Vorderer et al., 2004, p. 389). No obstante, su exploración en el ámbito de la tav es aún incipiente.

El entretenimiento está relacionado con el disfrute de la experiencia audiovisual aunque no son sinónimos. Ambos conceptos han sido analizados y teorizados en los estudios de comunicación (Vorderer et al., 2004, Soto-Sanfiel 2015). La teoría sostiene que el disfrute es el objetivo principal del espectador que busca el entretenimiento mediante el consumo de contenidos audiovisuales. El disfrute incluye varios tipos de experiencias psicológicas inducidas por una narrativa audiovisual que abarca las reacciones positivas a dichos contenidos (Vorderer et al., 2004, p. 388). El disfrute puede ser provocado por emociones positivas y negativas. También se ha demostrado que puede ser predicho por la sensación de estar inmerso en una narrativa y en particular por la transportación al mundo narrativo (Green \& Brock, 2000; Green, Strange \& Brock, 2002). Además, hay evidencias de que el disfrute es predicho por el compromiso narrativo (Soto-Sanfiel, 2015). Este también llamado enganche ${ }^{3}$ se define como la actuación conjunta de la transportación psicológica al mundo narrativo, la identificación con el personaje o la presencia en el espacio de la narrativa. Todos estos factores describen diferentes aspectos del compromiso narrativo que representa el grado de involucramiento de un espectador con la narrativa (Busselle \& Bilandzic, 2009).

Las experiencias fenomenológicas que contribuyen a la sensación de enganche han sido exploradas por diversos investigadores que también han construido y validado escalas para su medición (Witmer \& Singer, 1998; Green \& Brock, 2000; Biocca, 2002; Tal-Or \& Cohen, 2010). En la misma línea, los autores Buselle y Bilandzic (2009) desarrollaron una escala de medición del enganche en idioma inglés basada en los modelos mentales de procesamiento narrativo. Dicho modelo resultó de la agrupación de los componentes del fenómeno del enganche narrativo a los siguientes cuatro factores prominentes: comprensión narrativa, enfoque atencional, sensación de estar presente en la narrativa e involucramiento emocional con la narrativa ${ }^{4}$.

La comprensión narrativa está relacionada con el entendimiento de los personajes y el desarrollo del hilo conductor de la trama, por lo que no debería confundirse con la comprensión de los diálogos transmitidos también mediante el SPS. Del mismo modo, el enfoque atencional significa que existe motivación para ver un programa

3 Narrative engagement en la literatura original en inglés. En este trabajo se usan los términos compromiso narrativo y enganche para referirse al mismo concepto.

4 Los factores originales en inglés son los siguientes: narrative understanding (NU), attentional focus (AF), narrative presence (NP) y emotional involvement (EE).

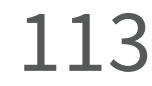




\section{DISERTACIONES}

ESTUDIOS

Estudios de audiencias y recepción: audiencias minoritarias y nuevas mediaciones

ISSN: 1856-9536

Doi: http://dx.doi.org/10.12804/revistas.urosario.edu.co/disertaciones/v11i1

Volumen 11, Número 1 / Enero-junio 2018

Versión PDF para imprimir desde

http://revistas.urosario.edu.co/index.php/disertaciones

sin interrupciones y obviar las posibles distracciones. La concentración es una condición del concepto de estar presente (Fontaine, 1992) o la sensación de "estar dentro" de la historia que, a su vez, suscita el disfrute (Lombard \& Ditton, 1997, p. 23). El involucramiento emocional potencia dicha sensación, aunque las emociones que emergen de una narrativa están también relacionadas con el grado de importancia que cada espectador asigna a un estímulo concreto (Witmer \& Singer, 1998, p. 227). Los sentimientos de empatía y simpatía hacia los protagonistas son los principales motivos para involucrarse emocionalmente con una narrativa (Busselle \& Bilandzic, 2009). Todos los conceptos mencionados y sus interacciones forman el tejido que representa el enganche a la experiencia audiovisual. Por consiguiente, es necesario estudiar estos factores si queremos conseguir que los servicios de accesibilidad permitan que el espectador disfrute la experiencia. En el caso del sPs, este objetivo se puede interpretar como una compensación visual de lo que se pierde por el canal acústico y contribuye a que el espectador se enganche con un programa y, en última instancia, pueda disfrutarlo.

\section{Aproximación e hipótesis de investigación}

Desde la entrada del sonido en el cine, se ha atribuido tradicionalmente más importancia al componente verbal, dado que el sonido es "voco- y verbocéntrico" (Chion, 1994, p. 6), sobre todo si nos referimos a la construcción de la trama. Por eso, se ha ignorado de manera general la contribución de los efectos sonoros a la hora de capturar la atención del espectador y estimular sentimientos. No obstante, los efectos sonoros son lo suficientemente versátiles como para contribuir en la construcción de la narrativa con sus varias funciones (Remael, 2012; Tsaousi, 2015). Además, los efectos sonoros tienen la capacidad de crear imágenes mentales (Rodero, 2014) o imágenes sonoras que aumentan el índice de credibilidad (Balsebre, 1994; Gutiérrez \& Perona, 2005). Este efecto ha sido relacionado también con el posible involucramiento con una narrativa (Green, 2004). Cabe destacar que los componentes de la comunicación audiovisual y del fenómeno de enganche de los espectadores funcionan en sinergia y por lo tanto aislar o asignar grados específicos de contribución a los componentes sonoros no corresponde con los propósitos de este estudio.

Por otro lado, los sonidos no verbales y su transmisión de manera visual en el sps son uno de los rasgos más distintivos de esta práctica. El parámetro extralingüístico, que incluye los efectos sonoros, la música y los elementos paralingüísticos, es el que diferencia el sps del subtitulado interlingüístico (Arnáiz-Uzquiza, 2012), dada la falta de acceso o el acceso limitado al sonido por parte de la audiencia. Los diversos grados de acceso al componente sonoro indican también la heterogeneidad que define la audiencia de sps (Neves, 2005). Dicha variedad en la caracterización de los receptores se refleja en la terminología empleada: sordos y personas con diversidad funcional auditiva apunta a la clasificación clínica según el tipo de audición medido en audiogramas. Muchas entidades reguladoras, tanto a nivel nacional como internacional, diferencian de esta forma entre deficiencia auditiva ligera, mediana, severa, profunda y cofosis (Biap $\left.{ }^{5}, 1997\right)$. Desde una perspectiva sociocultural, también se puede

5 Abreviación de Bureau International d’Audio Phonologie. A nivel nacional, la Confederación Estatal de Personas Sordas (CNSE) aplica la misma clasificación clínica. 


\section{DISERTACIONES}

ESTUDIOS

Estudios de audiencias y recepción: audiencias minoritarias y nuevas mediaciones

ISSN: 1856-9536

Doi: http://dx.doi.org/10.12804/revistas.urosario.edu.co/disertaciones/v11i1

Volumen 11, Número 1 / Enero-junio 2018

Versión PDF para imprimir desde

http://revistas.urosario.edu.co/index.php/disertaciones

diferenciar a las personas sordas 6 , es decir, las personas que pertenecen a la comunidad sorda y reivindican el uso de la lengua de signos. Esta clasificación basada en la modalidad de comunicación de los usuarios es la que se ha considerado más relevante en el campo de la tav (Matamala \& Orero, 2010). De este modo, se diferencia entre las personas sordas prelocutivas (que perdieron la audición antes de la edad de adquisición del lenguaje) y las personas sordas poslocutivas (que perdieron la audición después de dicha edad). Aun así, el uso de una lengua oral o una lengua de signos depende de muchos factores, como la educación recibida y el bagaje lingüístico y cultural de la familia, que varía según el usuario (Serrat-Manén, 2011).

La modalidad de la lengua usada indica la familiaridad con un sistema de representación acústico y verbal (lenguaje oral) o con un sistema visual no verbal -lenguaje de signos-(Taub, 2000; Pizzuto, 2007). Estudios previos afirman que los usuarios de lenguas de signos tienen una mayor capacidad para la representación gráfica (Cambra et al., 2010), la cual está relacionada con la atención visual de las personas sordas. En concreto, el campo visual de la personas sordas se distribuye más hacia la periferia, en comparación con la visión centralizada de los oyentes (Bavelier et al., 2006). Eso implica que a la hora de seguir un programa audiovisual subtitulado, los usuarios de lenguas de signos también se centran más en la información no verbal de la imagen que en los subtítulos (Miquel-Iriarte, 2014). En la misma línea, otros estudios demuestran que los mismos usuarios ignoran el área de los subtítulos (Arnáiz-Uzquiza, 2012) o si, por el contrario, deciden leer los subtítulos, dedican más tiempo a la lectura que a la imagen ${ }^{7}$ (Jensema et al., 2000; Szarkowska et al., 2011). Por eso, el uso de representaciones gráficas, como íconos o pictogramas, podría disminuir la información verbal en la pantalla y así reducir también la media de tiempo de lectura. Este hecho permitiría pasar más tiempo en la imagen y contribuiría a que la experiencia audiovisual fuera más positiva para este tipo de audiencias, lo que posiblemente aumentaría el disfrute total. Los primeros estudios realizados sobre la representación gráfica en el sPs para los efectos sonoros han aportado datos contradictorios entre los espectadores de diferentes países sobre sus preferencias al respecto (Romero-Fresco, 2015). Dichas contradicciones, aparte de confirmar la diversidad que se encuentra en estas poblaciones, también se pueden entender como el resultado de las diferentes prácticas de subtitulación que se usan en cada país, a las que están acostumbrados los espectadores.

En resumen, el perfil lingüístico y auditivo, y los hábitos a la hora de ver programas subtitulados son parámetros que influyen en la recepción. Dichos factores aumentan la heterogeneidad de la audiencia del sps que, a su vez, obstaculiza la labor de encontrar prácticas y estrategias que cubran necesidades tan diversas, como ha sido el objetivo de diversas entidades normalizadoras (AENOR 2012). Ante la ausencia de estudios que observen el efecto de la modalidad de representación en la experiencia narrativa, este trabajo pretende explorar las posibles diferencias entre los espectadores que usan mayoritariamente una o más lenguas de signos y aquellos que prefieren

6 De manera similar, en inglés existe el término Deafhood (con "D" mayúscula) que propuso Ladd (2003) como alternativa al término clínico. Este concepto se considera más positivo, ya que apunta a la cultura sorda en vez de centrarse en las limitaciones auditivas. Para una perspectiva más detallada del discurso, véase Kusters y de Meulder (2013). En España se emplea el término diversidad funcional (Romañach \& Lobato, 2005) como alternativa a términos negativos tales como «discapacidad".

7 El concepto de velocidad de visionado (viewing speed) forma parte de la terminología de la tav durante los últimos años para complementar el concepto de la velocidad de lectura de los subtítulos (Szarkowska et ál., 2011, Romero-Fresco, 2015). 


\section{DISERTACIONES}

ESTUDIOS

la comunicación oral. La literatura previa establece que los signantes tienen una mayor capacidad para la representación gráfica (Cambra et al., 2010) y, por lo tanto, la inclusión de recursos gráficos para los efectos sonoros (Civera \& Orero, 2010) podría tener algún efecto en el compromiso narrativo del espectador signante. Este efecto se contrasta con las personas que están acostumbradas a la representación verbal. En concreto, nuestro objetivo principal es aportar datos sobre el compromiso narrativo de personas con diversos perfiles lingüísticos y auditivos, mediante las hipótesis que se detallan a continuación:

H1: El tipo de estrategia de representación de los efectos sonoros (verbal o no verbal) afectará el enganche a la narrativa audiovisual dependiendo del perfil lingüístico de los espectadores.

H1a: Los usuarios de lenguas orales se verán más enganchados cuando los efectos sonoros se presentan con estrategias verbales.

H1b: Los usuarios de lenguas de signos se verán más enganchados cuando los efectos sonoros se presentan con estrategias no verbales.

Dada también la relación observada entre el enganche narrativo y el disfrute (Busselle \& Bilandzic, 2009; Soto-Sanfiel, 2015) y, en concreto, la capacidad del enganche a la hora de predecir el disfrute, el presente estudio también pretende confirmar, para esta muestra, la siguiente hipótesis:

$\mathrm{H} 2$ : El enganche es un predictor del disfrute.

\section{Método}

Se realizó un estudio cuasiexperimental con un diseño factorial $2 \times 2$ intersujetos con las variables: tipo de lenguaje usado (oral vs. de signos) y modalidad de la representación, es decir, estímulo verbal (descripción del sonido) vs. no verbal (íconos). La asignación de los participantes a los grupos de recepción fue aleatoria. Los análisis estadísticos se realizaron con los programas spss 21 y amos 23.

\section{Participantes}

Los participantes se reclutaron a través de asociaciones de Cataluña, contactos personales y la pagina web ${ }^{8}$ creada con el mismo propósito. Un total de 46 personas participaron voluntariamente en las sesiones sin recibir ningún tipo de compensación económica. El número de participantes coincide con el número total de respuestas obtenidas. El 58,7\% de los participantes fueron mujeres y el 41,3\% fueron hombres. La media de edad de la muestra fue de 58,7 años (Rg = 19-83, DT =2,4). En cuanto a su perfil lingüístico, 21 participantes (45,7\%) reportaron usar una o más lenguas orales (catalán, castellano o ambas) y 25 (54,3\%) una o más lenguas de signos (sobre todo la lengua de signos catalana). En cuanto a sus hábitos como espectadores, 32 participantes $(69,5 \%)$ afirman que hacen uso habitual de los subtítulos para seguir un programa, mientras que el restante $30,4 \%$ no usa ningún tipo de servicio para facilitar su acceso al canal auditivo.

8 www.cinevisual4all.com 


\section{Procedimiento}

Los participantes acudían en grupos de entre cinco a diez personas a una sala en la que estaba dispuesto un ordenador y un proyector. Al llegar, eran informados del procedimiento con una breve introducción sobre el estudio acompañada por la hoja informativa y el consentimiento ético que debían firmar ${ }^{9}$. Seguidamente, los participantes rellenaban un cuestionario pretratamiento con preguntas generales (género, edad, uso de lenguaje y hábitos como espectador). A continuación, se proyectaba la película (8 min.) creada específicamente para los propósitos del estudio. Tras el visionado, los participantes completaban un cuestionario postratamiento. La duración de la prueba no superó los 60 minutos en ninguna de las sesiones.

\section{Materiales}

Para incluir todas las funciones posibles del mismo efecto sonoro (Tsaousi, 2015) en diferentes contextos se decidió crear un cortometraje ${ }^{10}$ que permitiese una duración adecuada del proceso experimental con los participantes (que contase una historia completa, que permitiese generar enganche y que, al mismo tiempo, no tuviese una duración excesiva para no cansar a los participantes). El cortometraje al que posteriormente se añadieron los subtítulos e íconos se rodó en la Universitat Autònoma de Barcelona con la ayuda de voluntarios de la misma universidad. Se prestó especial atención al seguimiento de una estructura de trama tradicional de tres partes (Chion, 1992; Martínez-Sierra, 2012, p. 148), que permitiera el efecto deseado de enganche de los espectadores. A este efecto ayudó el género del cortometraje, ya que el drama puede provocar la empatía y la simpatía de la audiencia hacia los protagonistas. En concreto, la película trata de una pareja joven que decide separarse por motivos de distancia. La trama se centra en las reacciones de la protagonista (Claudia) que no parece estar dispuesta a aceptar esta nueva realidad. Para los cuatro casos del efecto sonoro, se eligió el sonido del teléfono, dado que es un elemento fácil de insertar en una trama y también por ser un objeto muy familiar para todas las audiencias. El estímulo se dividió en dos grupos, el primero vio la película con la estrategia verbal y el segundo con la estrategia no verbal (tabla 1).

9 Ambos documentos fueron aprobados previamente por la Comisión de Ética en la Experimentación Animal y Humana (сеEAH) de la Universitat Autònoma de Barcelona.

10 Un efecto positivo de esta decisión fue el hecho de no necesitar permisos sobre los derechos de autor. Incluso dentro de los propósitos académicos, manipular y diseminar materiales audiovisuales es un problema recurrente en el campo de TAv. 


\section{DISERTACIONES}

Estudios de audiencias y recepción: audiencias minoritarias y nuevas mediaciones ISSN: 1856-9536

Doi: http://dx.doi.org/10.12804/revistas.urosario.edu.co/disertaciones/v11i1 Volumen 11, Número 1/ Enero-junio 2018

Versión PDF para imprimir desde

http://revistas.urosario.edu.co/index.php/disertaciones

Tabla 1. Instancias del sonido de teléfono

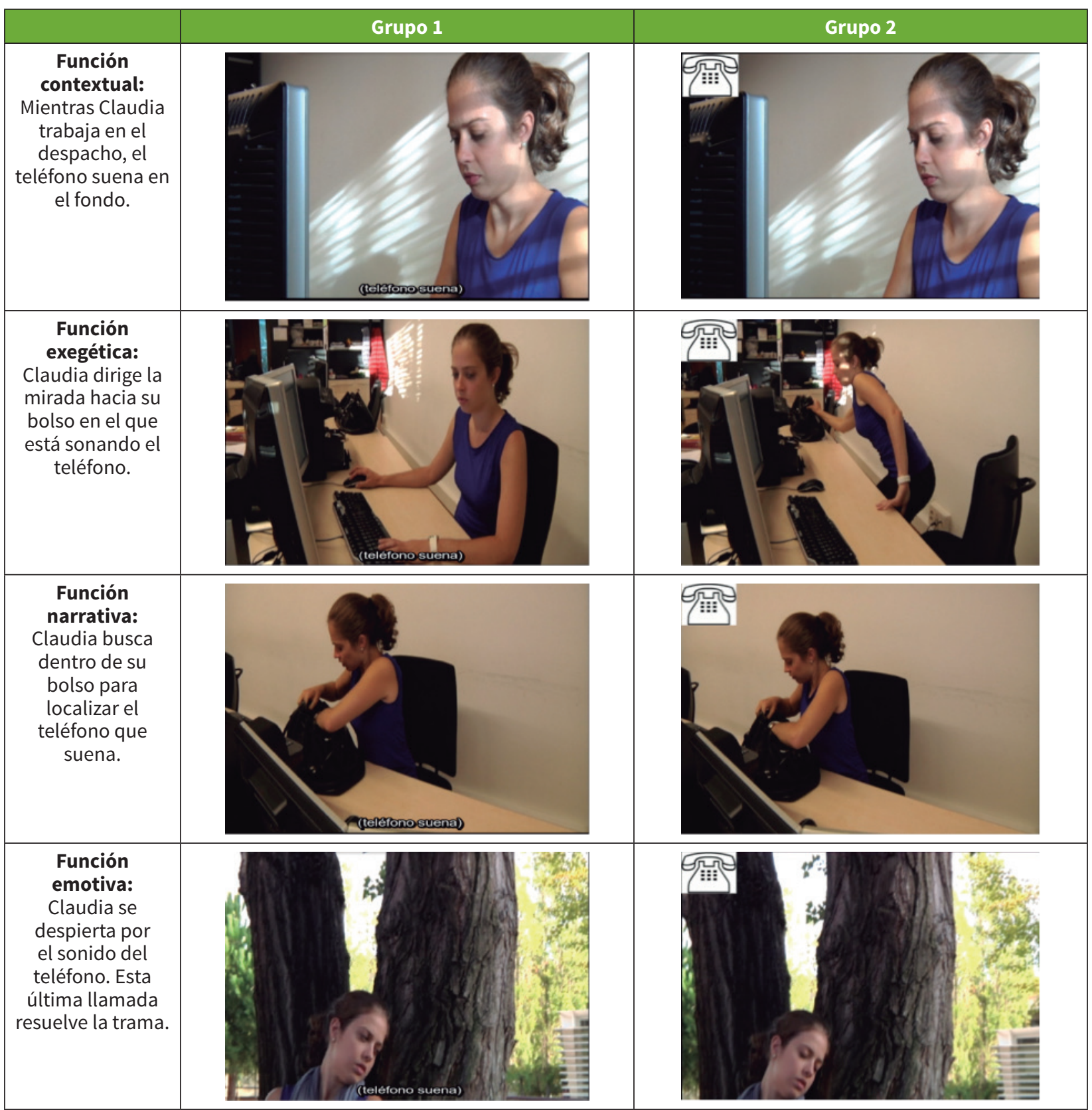

Inmediatamente después del visionado, los participantes respondieron la escala de enganche propuesta por Busselle y Bilandzic (2009) que fue elegida por medir la totalidad de los aspectos que componen el compromiso 


\section{DISERTACIONES}

ESTUDIOS

Estudios de audiencias y recepción: audiencias minoritarias y nuevas mediaciones

ISSN: 1856-9536

Doi: http://dx.doi.org/10.12804/revistas.urosario.edu.co/disertaciones/v11i1

Volumen 11, Número 1 / Enero-junio 2018

Versión PDF para imprimir desde

http://revistas.urosario.edu.co/index.php/disertaciones

narrativo ${ }^{11}$. La escala original en inglés se tradujo al castellano para esta investigación y se modificó el ítem "No llegué a comprender el modo de pensar de los protagonistas" por el ítem "No llegué a comprender el modo de pensar de Claudia”, dado que es el único personaje que se desarrolla en la trama. La escala final se compuso de 12 ítems, igual que la escala original. La valoración de los ítems era de cinco puntos $(1=$ totalmente en desacuerdo $/ 5=$ totalmente de acuerdo). Dichos ítems representaban los cuatro bloques medidos por la escala de la siguiente forma:

- Comprensión narrativa (3 ítems: ENG1, ENG2, ENG4)

- Enfoque atencional (3 ítems: ENG5, ENG6, ENG7)

- Presencia narrativa (3 ítems: ENG8, ENG9, ENG10)

- Involucramiento emocional (3 ítems: ENG11, ENG12, ENG13)

Por último, se añadieron tres ítems creados para esta investigación basados en Soto-Sanfiel et al. (2009) para medir el disfrute ("He disfrutado la película"), la satisfacción ("Me ha gustado la película") y la disposición de repetir la experiencia ("No volvería a ver una historia semejante") con la misma escala de valoración ( 1 = totalmente en desacuerdo / 5 = totalmente de acuerdo).

\section{Resultados}

El primer paso fue validar la escala original, que obtuvo un índice de fiabilidad aceptable $(\alpha=0,639)$ para la muestra (Cortina, 1993). Posteriormente realizamos un análisis factorial exploratorio en el que se usó la rotación varimax y extracción de los componentes principales. El análisis mostró la existencia de los cuatro componentes del modelo original propuesto (Busselle \& Bilandzic, 2009), que explicaron el 47\% de la varianza en la validación original. En el presente caso, sin embargo, los mismos componentes explicaron el 69,20\% de la varianza (tabla 2).

Tabla 2: Análisis factorial con rotación varimax y normalización Kaiser para el compromiso narrativo

\begin{tabular}{|c|c|c|c|c|}
\hline & \multicolumn{4}{|c|}{ Componente } \\
\hline & 1 & 2 & 3 & 4 \\
\hline ENG1: A veces me costaba entender lo que pasaba en la película. & & & & ,833 \\
\hline ENG2: No llegué a comprender el modo de pensar de Claudia. & & & &, 572 \\
\hline ENG4: Me costó seguir el argumento. & & & & ,757 \\
\hline ENG5: Mientras veía la película estaba pensando en otras cosas. & & & 849 & \\
\hline ENG6: Me costó concentrarme en la película. & & & 813 & \\
\hline ENG7: Estaba más atento/a en lo que pasaba a mi alrededor que en la película. & & & ,766 & \\
\hline $\begin{array}{l}\text { ENG8: Durante la proyección, estaba físicamente en el aula, pero mentalmente } \\
\text { estaba en el mundo paralelo de la película. }\end{array}$ & &, 509 & & \\
\hline ENG9: La película creó un mundo que desapareció de repente cuando esta se acabó. & & 713 & & \\
\hline $\begin{array}{l}\text { ENG10: A veces, durante la proyección, me vi más involucrado/a en el mundo de la } \\
\text { película que en el mundo real. }\end{array}$ & & ,743 & & \\
\hline
\end{tabular}

11 Las escalas desarrolladas por otros autores (Green et al., 2004) miden conceptos correlacionados pero no el fenómeno del compromiso narrativo en su conjunto. 


\section{DISERTACIONES}

Estudios de audiencias y recepción: audiencias minoritarias y nuevas mediaciones

ISSN: 1856-9536

Doi: http://dx.doi.org/10.12804/revistas.urosario.edu.co/disertaciones/v11i1

Volumen 11, Número 1 / Enero-junio 2018

Versión PDF para imprimir desde

\begin{tabular}{|l|c|c|c|c|}
\hline & \multicolumn{3}{|c|}{ Componente } \\
\cline { 2 - 5 } & $\mathbf{1}$ & $\mathbf{2}$ & $\mathbf{3}$ & $\mathbf{4}$ \\
\hline ENG11: La película me impactó emocionalmente. &, 797 & & & \\
\hline $\begin{array}{l}\text { ENG12: Durante la proyección, cuando los protagonistas estaban felices, me sentía } \\
\text { contento/a y cuando ellos sufrían lo pasaba mal. }\end{array}$ &, 741 & & & \\
\hline ENG13: Sentí pena por los protagonistas. &, 852 & & & \\
\hline
\end{tabular}

Tras comprobar que los factores se ajustaban al modelo original, se realizó un análisis factorial confirmatorio (AFC - tabla 3) con la matriz de correlaciones policóricas (Jorerskog \& Sorbom, 1979) y el método de estimación Unweighted Least Squares (uss), que es el indicado para muestras pequeñas con datos ordinales y categóricos. Mediante la matriz de correlaciones policóricas no se detectó multicolinealidad. En dicho análisis detectamos los siguientes valores de índices globales de ajuste: $\chi^{2}=0,53, \mathrm{CFI}=1,39$, RMSEA $=0,094$ y SRMR $=0,08$. Estos índices permiten valorar el ajuste como admisible en nuestro caso, dado el tamaño de la muestra y el número de ítems (Jackson et al., 2009).

En cuanto a las interacciones entre los componentes principales, se observan correlaciones positivas significativas $(p<0,01)$ entre los factores: comprensión narrativa y enfoque atencional $(r=0,398)$, así como en los factores: involucramiento emocional y presencia narrativa $(r=0,478)$. En cuanto a los factores añadidos a la escala original: el disfrute ( $M=3,80, D T=1,142$ ), la disposición ( $M=2,63$, $D T=1,142$ ) y la satisfacción ( $M=3,67, D T=1,076)$, se muestra una correlación positiva entre el disfrute y la satisfacción $(p<0,01, r=0,674)$, el disfrute y el involucramiento emocional $(p=0,001, r=0,493)$ y también entre el disfrute y la presencia narrativa $(p=0,001, r=0,528)$. Por último, se puede detectar una correlación significativa entre satisfacción y presencia narrativa $(p=0,004, r=$ $0,415)$. Estos resultados nos permiten confirmar la $\mathrm{H} 2$ dadas las correlaciones observadas entre el disfrute y los componentes de enganche. 


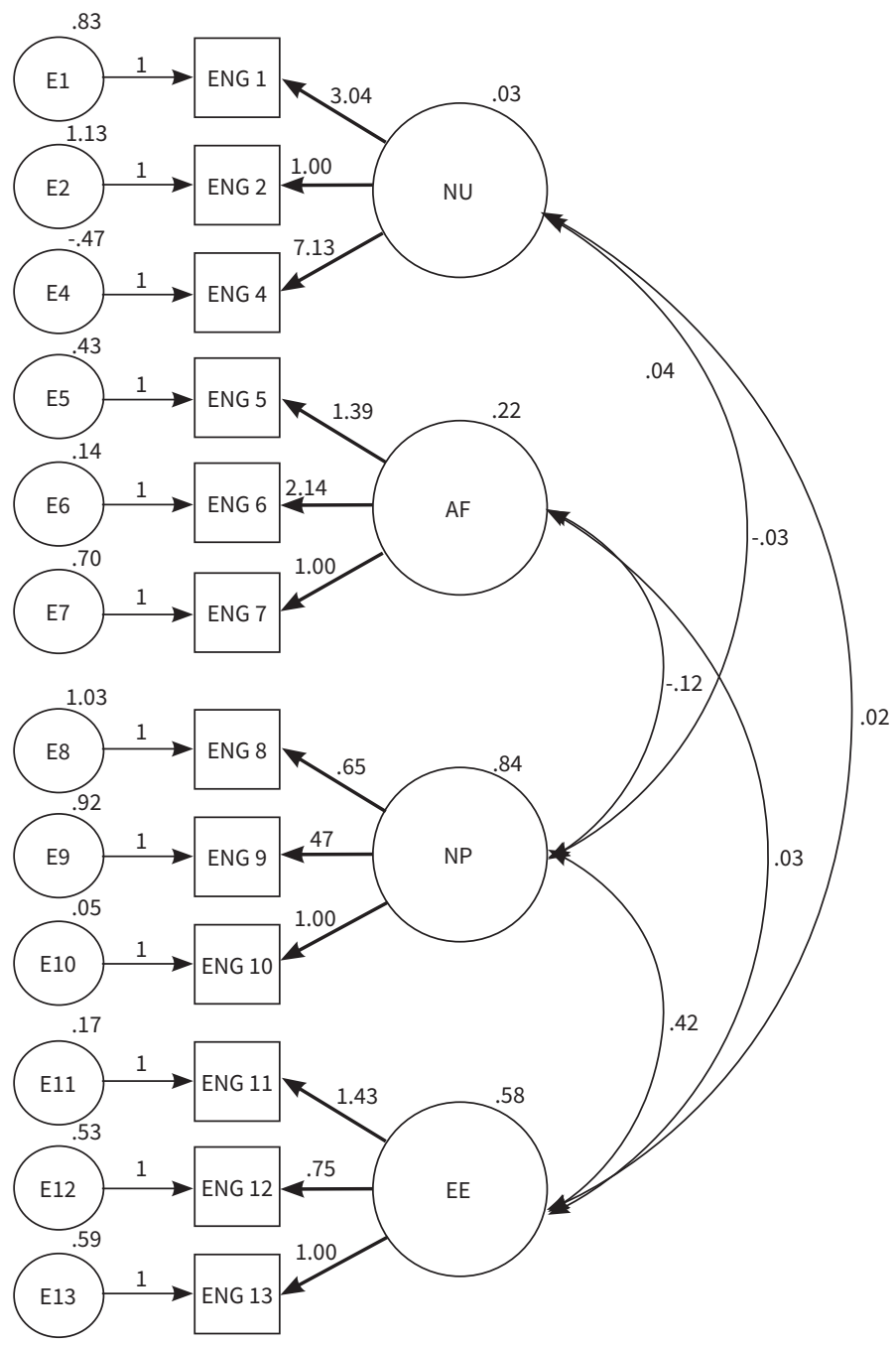

Figura 1. Análisis confirmatorio de cuatro factores

En las pruebas no paramétricas de U Mann-Whitney para muestras independientes entre los participantes de perfil oralista $(N=21)$ y los participantes de perfil signante $(N=25)$ no se observaron diferencias significativas en ninguno de los factores principales. En concreto, no hubo diferencias entre el primer y el segundo grupo en las medidas de disfrute, la disposición de repetir la experiencia ni en los cuatro factores de enganche. Lo mismo ocurrió con la comparación entre los grupos que vieron la película con el estímulo verbal $(\mathrm{N}=20)$ y los que la vieron con el estímulo no verbal $(\mathrm{N}=26)$, es decir $\mathrm{p}=0,05 \mathrm{y} \mathrm{U}=205-230$, por lo cual podemos rechazar tanto la hipótesis $\mathrm{H} 1$ como las hipótesis H1a y H1b. En conclusión, el tipo de estímulo para la representación de los efectos sonoros no afecta el disfrute ni el enganche de la experiencia audiovisual, la cual es muy parecida tanto para las personas que acostumbran a comunicarse mediante una lengua de signos como para las que usan mayoritaria o exclusivamente lenguas orales. 


\section{DISERTACIONES}

ESTUDIOS

Estudios de audiencias y recepción: audiencias minoritarias y nuevas mediaciones

ISSN: 1856-9536

Doi: http://dx.doi.org/10.12804/revistas.urosario.edu.co/disertaciones/v11i1

Volumen 11, Número 1 / Enero-junio 2018

Versión PDF para imprimir desde

http://revistas.urosario.edu.co/index.php/disertaciones

\section{Conclusiones}

Hasta la fecha los estudios de recepción en el sps se han centrado mayoritariamente en las preferencias de la audiencia y en la percepción visual de los subtítulos e imágenes. Este estudio se añade a los que exploran el componente sonoro, no verbal en relación al sps y a los que se enfocan en los espectadores de la misma práctica (Romero-Fresco, 2015). Además, es un primer intento de incorporar los conceptos involucrados en la experiencia audiovisual, tales como el disfrute y el enganche, en las aproximaciones empíricas al sps. Dichos conceptos, explorados en los estudios de comunicación, demuestran estar relacionados también con la muestra sorda y con diversidad funcional auditiva que participó en el presente estudio. Así, esta investigación ha comprobado el modelo propuesto por Busselle y Bilandzic (2009) para la medición del enganche, al igual que la interacción entre los componentes del mismo y el disfrute de la experiencia audiovisual, que es uno de los objetivos del entretenimiento. En concreto, el disfrute se relacionó con los factores de involucramiento emocional de los espectadores y con la sensación de estar presente en la narrativa. Cabe destacar, sin embargo, que los resultados que aquí se recogen representan una muestra limitada, aunque bastante heterogénea a nivel de edad y de perfiles lingüísticos y auditivos.

Por otro lado, este estudio ha demostrado que las representaciones verbales o no verbales para los efectos sonoros no afectan el enganche ni el disfrute de una película, los cuales son muy parecidos tanto para las audiencias acostumbradas a la comunicación no verbal mediante el uso de lenguas de signos, como para los espectadores que se comunican sobre todo por vía verbal mediante una lengua oral. Este resultado indica que el tipo de representación de los efectos sonoros no tiene ningún efecto significativo en la experiencia audiovisual para esta muestra. Una explicación posible de este dato es que la audiencia está acostumbrada a las representaciones verbales usadas actualmente en los canales televisivos españoles y, por lo tanto, su presencia en la pantalla no tiene un efecto notable. Al contrario, las representaciones gráficas no se usan habitualmente y por eso la audiencia puede necesitar un período de adaptación para obtener respuestas más positivas. Sin embargo, sería interesante replicar este estudio eliminando por completo la información sobre los efectos sonoros para poder comprobar si eso se debe al papel secundario que se asigna a los efectos sonoros en general. De manera similar, se podría comprobar si la transmisión de los diálogos por medio de interpretación de lengua de signos podría cambiar este resultado y sugerir diferencias significativas entre ambos grupos. La modalidad de presentación del cuestionario, que en este caso ha sido verbal también podría afectar la comprensión del mismo. Por lo tanto, se podría emplear también un cuestionario electrónico en lengua de signos. Además, este estudio podría complementarse con datos sobre la percepción visual de los espectadores y, concretamente, del tiempo que los espectadores dedican a la imagen con estrategias verbales y con estrategias no verbales y otras medidas psicofisiológicas y mixtas, tanto cualitativas como cuantitativas.

En resumen, este estudio arroja importante información para el sPS, que tiene como objetivo principal que los espectadores que usan dicho servicio puedan tener acceso a la información sonora y disfrutar de un programa audiovisual. Como se demostró, el tipo de representación no afecta la experiencia narrativa, por lo cual las representaciones no verbales podrían ser igual de eficaces y utilizarse más en el futuro. Otro factor a investigar sería si dichas estrategias tienen un efecto similar a nivel de esfuerzo cognitivo. Además, este estudio podría ser replicado con otros contenidos, dado que el género audiovisual puede afectar el enganche según se ha comprobado en un 


\section{DISERTACIONES}

ESTUDIOS

Estudios de audiencias y recepción: audiencias minoritarias y nuevas mediaciones

ISSN: 1856-9536

Doi: http://dx.doi.org/10.12804/revistas.urosario.edu.co/disertaciones/v11i1

Volumen 11, Número 1 / Enero-junio 2018

Versión PDF para imprimir desde

http://revistas.urosario.edu.co/index.php/disertaciones

estudio previo (Soto-Sanfiel, 2015). Por último, el estudio podría ampliarse con muestras de otros contextos regionales y diversos perfiles lingüísticos y culturales.

\section{Referencias}

1. Asociación Española de Normalización y Certificación. (2012). Norma unE 153010: subtitulado para personas sordas y personas con discapacidad auditiva. subtitulado a través del teletexto. Madrid: AENOR.

2. Arnáiz-Uzquiza, V. (2012). Los parámetros que identifican el subtitulado para sordos. Análisis y clasificación. En R. Agost, P. Orero \& E. di Giovanni (Eds.), Multidisciplinarity in Audiovisual Translation (pp. 103132). Alacant: Universitat d'Alacant.

3. Arnáiz-Uzquiza, V. (2015). Long questionnaire in Spain. En P. Romero-Fresco (Ed.). The Reception of SDH in Europe (pp. 95-116). Bern: Peter Lang.

4. Balsebre, A. (1994). El lenguaje radiofónico. Madrid: Ediciones Cátedra.

5. Bavelier, D., Dye, M., \& Hauser, P. (2006). Do deaf individuals see better? Trends in Cognitive Science, 10, 512-518.

6. Bureau International d'Audio Phonologie. (1997). Classification audiométrique des déficiences auditives. Lisboa. Recuperado de https://www.biap.org/es/archivo/65-ct-2-classification-des-surdites

7. Biocca, F. (2002). The evolution of narrative towards "being there" in non-linear narrative worlds. En M. Green, J. Strange \& T. Brock (Eds.). Narrative impact: Social and cognitive foundations (pp. 2-47). Hillsdale: Lawrence Erlbaum.

8. Busselle, R., \& Bilandzic, H. (2009). Measuring narrative engagement. Media Psychology, 12(4), 321-347.

9. Cambra, C., Leal, A., \& Silvestre, N. (2008). Función de la subtitulación y la interpretación de la imagen en la comprensión de los mensajes televisivos: la comprensión de una serie por parte de los adolescentes sordos. Cultura y Educación, 20(1), 81-93.

10. Cambra, C., Leal, A., \& Silvestre, N. (2009). Comprehension of television messages by deaf students at various stages of education. American Annals of the Deaf, 153(5), 425-434.

11. Cambra, C., Leal, A., \& Silvestre, N. (2010). How deaf and hearing adolescents comprehend a televised story. Deafness and Education International, 12(1), 34-51.

12. Chion, M. (1992). Como se escribe un guión. Madrid: Cátedra.

13. Chion, M. (1994). Audio-Vision: Sound on screen. Nueva York: Columbia University Press.

14. Civera, C., \& Orero, P. (2010). Introducing icons in subtitling for the deaf and hard of hearing: Optimising reception?. En A. Matamala y P. Orero (Eds.), Listening to subtitles: Subtitles for the deaf and hard of hearing (pp. 149-161). Berna: Peter Lang.

15. Cortina, J. M. (1993). What is coefficient alpha? An examination of theory and applications. Journal of Applied Psychology, 78, 98-104.

16. Fontaine, G. (1992). The experience of a sense of presences in intercultural and international encounters. Presence: Teleoperators and virtual environments, 1(4), 482-490.

17. Fryer, L., \& Freeman, J. (2012). Presence in those with and without sight: implications for virtual reality and audio description. Journal of Cybertherapy and rehabilitation, 5(1), 15-23. 


\section{DISERTACIONES}

ESTUDIOS

Estudios de audiencias y recepción: audiencias minoritarias y nuevas mediaciones

ISSN: 1856-9536

Doi: http://dx.doi.org/10.12804/revistas.urosario.edu.co/disertaciones/v11i1

Volumen 11, Número 1 / Enero-junio 2018

Versión PDF para imprimir desde

http://revistas.urosario.edu.co/index.php/disertaciones

18. Gambier, Y. (2006). Multimodality and audiovisual translation. En Audiovisual translation scenarios: conference proceedings. EU-High-Level Scientific Conference Series. Recuperado de http://www.euroconferences.info/proceedings/2006_Proceedings/2006_Gambier_Yves.pdf

19. Gottlieb, H. (2005). Multidimensional translation: Semantics turned semiotics. MuTra 2005, Challenges of Multidimensional Translation: Conference Proceedings.

20. Green, M. (2004). Transportation into narrative worlds: The role of prior knowledge and perceived realism. Discourse Processes, 38(2), 247-266.

21. Green, M., \& Brock, T. (2000). The role of transportation in the persuasiveness of públic narratives. Journal of Personality and Social Psychology, 79, 701-721.

22. Green, M., Strange, J., \& Brock, T. (Eds.). (2002). Narrative impact: social and cognitive foundations. Hillsdale: Lawrence Erlbaum.

23. Green, M., Brock, T., \& Kaufman, G. (2004). Understanding media enjoyment: The role of transportation into narrative worlds. Communication Theory, 14, 311-327.

24. Gutiérrez García, M., \& Perona Páez, J.J. (2005). Teoría y técnica del lenguaje radiofónico. Barcelona: Bosch.

25. Henerson, M., Morris, L.L., \& Firz-Gibbon, C. (1987). How to measure attitudes. Beverly Hills: Sage Publishing.

26. Jackson, D., Gillaspy A., \& Purc-Stephenson, R. (2009). Reporting practices in confirmatory factor analysis: An overview and some recommendations. Psychological Methods, 14(1), 6-23.

27. Jennet, C., Cox, A., Cairns, P., Dhoparee, S., Epps, A., Tijs, T., \& Walton, A. (2008). Measuring and defining the experience of immersion in games. International Journal of Human-Computer Studies, 66(9), 641-661.

28. Jensema, C., Sharkawy, S., Danturthi, R.S., Burch, R., \& Hsu, D. (2000). Eye movements patterns of captioned television viewers. American Annals of the Deaf, 145(3), 275-285.

29. Jöreskog, K. G., \& Sörbom, D. (1979). Advances in factor analysis and structural equation models. Nueva York: University Press of America.

30. Kirkland, E. (1999). Evaluating of captioning features to inform development of digital television captioning facilities. American Annals of the Deaf, 144 (3), 250-260.

31. Kruger, J. L., \& Steyn, F. (2014). Subtitles and eye tracking: Reading and performance. Reading Research Quarterly, 49(1), 105-120.

32. Kruger, J. L., Szarkowska, A., \& I. Krejtz. (2015). Subtitles on the moving image: An overview if eye tracking studies. Journal of Entertainment Media. Recuperado de http://refractory.unimelb.edu.au/2015/02/07/ kruger-szarkowska-krejtz/v

33. Kruger, J.L., Doherty, S., \& Soto-Sanfiel, M.T. (2017). Original language subtitles: Their effects on the native and foreign viewer. Comunicar, 50, 23-32.

34. Kusters, A., \& De Meulder, M. (2013). Understanding deafhood: In search of its meanings. American Annals of the Deaf, 158(5), 428-438.

35. Ladd, P. (2003). Understanding Deaf Culture: In Search of Deafhood. Clevedon: Cromwell Press.

36. Lombard, M., \& Ditton, T. (1997). At the heart of it all: The concept of presence. The Journal of Computer Mediated Communication, 3(2). Recuperado de http://jcmc.indiana.edu/vol3/issue2/lombard.html

37. Lorenzo, L. (2010). Subtitling for deaf and hard of hearing children in Spain. A case study. En A. Matamal, \& P. Orero (Eds.), Listening to subtitles: Subtitles for the deaf and hard of hearing (pp. 115-138). Berna: Peter Lang. 


\section{DISERTACIONES}

ESTUDIOS

Estudios de audiencias y recepción: audiencias minoritarias y nuevas mediaciones

ISSN: 1856-9536

Doi: http://dx.doi.org/10.12804/revistas.urosario.edu.co/disertaciones/v11i1

Volumen 11, Número 1 / Enero-junio 2018

Versión PDF para imprimir desde

http://revistas.urosario.edu.co/index.php/disertaciones

38. Matamala, A., \& Orero, P. (Eds.). (2010). Listening to subtitles: Subtitles for the deaf and hard of hearing. Berna: Peter Lang.

39. Martínez Sierra, J. J. (2012). On the relevance of script writing basics in audiovisual translation practice and training. Cadernos de Tradução, 29(1), 154-163.

40. Miquel-Iriarte, M. (2014). The reception of subtitling by the deaf and hard of hearing. Preliminary findings. En E. Torres-Simon, \& D. Orrego-Carmona (Eds.), Translation research projects 5 (pp. 63-76). Tarragona: Intercultural Studies Group.

41. Neves, J. (2005). Audiovisual translation: Subtitling for the deaf and hard-of-hearing (Tesis doctoral, Londres: University of Surrey-Roehampton). Recuperado de http://roehampton.openrepository.com/roehampton/bitstream/10142/12580/1/neves\%2520audiovisual.pdf

42. Neves, J. (2008). 10 fallacies about Subtitling for the d/Deaf and the hard of hearing. The Journal of Specialised Translation 10.Recuperado de http://www.jostrans.org/issue10/art_neves.php

43. Orero, P., \& Vilaró, A. (2012). Eye tracking analysis of minor details in films for audio description. En R. Agost, P. Orero, \& E. di Giovanni (Eds.), Multidisciplinarity in audiovisual translation (pp. 295-319). Alacant: Universitat d'Alacant.

44. Perego, E. (2012). Eye-tracking in audiovisual translation. Roma: Aracne Editrice.

45. Pérez-González, L. (2014). Audiovisual translation. Londres: Routledge.

46. Rajendran , D., Duchowski, A., Orero, P., Martínez, J., \& Romero Fresco, P. (2013). Effects of text chunking on subtitling: A quantitative and qualitative examination. Perspectives: Studies in Translatology, 21(1), 5-21.

47. Ramos, M. (2015). The emotional experience of films: Does audio description make a difference? Translator, 21(1), 68-94.

48. Redmond, S. (2015). Eye tracking the sublime in spectacular moments of science fiction film. En S. Redmond, \& E. Marvell (Eds.). AFI film reader: Endangering science fiction film (pp. 32-50). Nueva York: Routledge.

49. Remael, A. (2012). For the use of Sound. Film sound analysis for audio-description: Some key issues." En R. Agost, P. Orero, \& E. di Giovanni (Eds.), Multidisciplinarity in Audiovisual Translation. Monografías de Traducción e Interpretación MonTI 4 (pp. 103-132). Alicante: Universidad de Alicante.

50. Rodero, E. (2012). See it on a radio story. Sound effects and shots to evoked imagery and attention on audio fiction. Communication Research, 39(4), 458-479.

51. Romañach, J., \& Lobato, M. (2005). Functional diversity, a new term in the struggle for dignity in the diversity of the human being. Independent Living Forum. Recuperado de http://disability-studies.leeds.ac.uk/ files/library/zavier-Functional-Diversity-Romanach.pdf

52. Romero-Fresco, P. (ed.). (2015). The reception of subtitles for the deaf and hard of hearing in Europe. Bern: Peter Lang.

53. Pizzuto, E., Pietandrea, P., \& Raffeele, S. (2007). Verbal and signed languages. Comparing structures, constructs and methodologies. Berlin: Mouton de Gruyter.

54. Pietrandrea, P., \& Russo, T. (2007). Diagrammatic and imagic hypoicons in signed and verbal languages. En E. Pizzuto, P. Pietrandrea, \& R. Simone (Eds.), Verbal and signed languages. Comparing structures, constructs and methodologies (pp. 35-56). Berlín: Mouton de Gruyter. 
55. Serrat-Manén, J. (2011). La percepció que tenen les persones sordes signants de l'actualitat periodística. exploració comparativa entre els estudiants de la gallaudet university (EUA) i la comunitat sorda catalana. Tesis doctoral. Barcelona: Universitat Autònoma de Barcelona.

56. Soto-Sanfiel, M. T., L. Aymerich-Franch, \& Ribes- Guàrdia, X. (2009). Interactividad y contenido como factores de disfrute en las ficciones interactivas. Revista Latina de Comunicación Social, 64, 668-681.

57. Soto-Sanfiel, M. T. (2015). Engagement and mobile listening. International Journal of Mobile Communications, 13(1), 29-50.

58. Szarkowska, A., Krejtz, I., Klyszejko, Z., \& Wieczorek, A. (2011). Verbatim, standard, or edited? Reading patterns of different captioning styles among deaf, hard of hearing, and hearing viewers. American Annals of the Deaf, 156(4), 363-378.

59. Tal-Or, N., \& Cohen, J. (2010). Understanding audience involvement: Conceptualizing and manipulating identification and transportation. Poetics, 38(4), 401-418.

60. Taub, S. F. (2000). Iconicity in American sign language: Concrete and metaphorical applications. Spatial Cognition and Computation, 2(1), 31-50.

61. Tsaousi, A. (2015). Making sound accessible: The labelling of sound effects in subtitling for the deaf and hard-of-hearing. Hermeneus, 17, 233-252.

62. Vorderer, P., Klimmt, C. y Ritterfeld, U. (2004). Enjoyment: At the heart of media entertainment. Communication Theory, 14(4), 388-408.

63. Witmer, B. \& Singer, M. (1998). Measuring presence in virtual environments: A presence questionnaire. Presence: Teleoperators and Virtual Environments, 7(3), 225-240. 